\title{
Évaluation de la classification infirmière des malades aux urgences (CIMU) : généralisation des résultats
}

\author{
Evaluation of the French Emergency Nurses Classification: Generalization of the Results
}

\author{
P.-G. Claret $\cdot$ X. Bobbia $\cdot$ J.-E. de La Coussaye
}

Reçu le 12 avril 2015; accepté le 15 avril 2015

(C) SFMU et Lavoisier SAS 2015

Le triage est nécessaire lorsque les soignants ne peuvent répondre immédiatement aux besoins des patients. Il est défini comme la hiérarchisation des prises en charge des patients en fonction de leur gravité. Cette définition implique des conséquences individuelles et collectives au triage. En effet, les objectifs du triage sont multiples. Ils sont de prendre en charge au mieux chaque patient, mais aussi d'organiser efficacement le système de soins. Un sous-triage entraîne une perte de chance et peut aggraver le pronostic du patient. Un sur-triage consomme des ressources souvent limitées, qui ne pourront être disponibles pour les autres patients. Le triage doit donc être réalisé à l'aide d'échelles spécifiques, par des professionnels expérimentés, mais aussi formés. Le triage en structure des urgences (SU) a fait l'objet de recommandations en 2012 sous l'égide de la Société française de médecine d'urgence [1]. Bien que des échelles de triage à trois niveaux soient encore utilisées [2], les échelles à cinq niveaux comme la classification infirmière des malades aux urgences (CIMU) semblent supérieures en termes de reproductibilité et d'exactitude [3-5].

La qualité d'une échelle de triage dépend de sa reproductibilité et de son exactitude. La reproductibilité est une mesure des erreurs aléatoires entre l'objectif défini et le niveau de triage. La reproductibilité peut être mesurée grâce aux pourcentages de concordance ou au coefficient kappa. L'exactitude est une mesure des erreurs systématiques entre l'objectif défini et le niveau de triage. L'exactitude peut être mesurée grâce aux coefficients de corrélation ou aux courbes ROC. La définition de cet objectif de référence, censée

P.-G. Claret $(\bowtie) \cdot$ X. Bobbia $\cdot$ J.-E. de La Coussaye Pôle anesthésie réanimation douleur urgences, centre hospitalier universitaire de Nîmes, 4 rue du Professeur Robert Debré, F-30029 Nîmes, France

e-mail : pierre.geraud.claret@gmail.com

J.-E. de La Coussaye

Université Montpellier-Nîmes, 2 rue École de Médecine,

F-34060 Montpellier, France représenter l'état de santé exact du patient, pose problème. Cet objectif de référence n'existant pas, il faut utiliser un critère de substitution. Peuvent être choisis comme critère de substitution le taux d'hospitalisation, la consommation de ressources, le taux d'admission en réanimation et soins intensifs ou encore la durée de séjour en SU. L'évaluation d'une échelle de triage est donc complexe, la reproductibilité et l'exactitude dépendant de l'objectif de l'échelle, mais aussi du personnel qui l'utilise.

Dans ce numéro des Annales françaises de médecine d'urgence, l'équipe de l'hôpital Lariboisière, Assistance publique - Hôpitaux de Paris (AP-HP), nous présente une étude dont l'objectif est d'évaluer la CIMU, en particulier la reproductibilité entre le triage des infirmier(ière)s organisateur(trice)s de l'accueil et celui des investigateurs [6]. Les auteurs retrouvent une bonne reproductibilité, évaluée avec un kappa de 0,64 (ICs $95 \%$ [0,61-0,67]). Dans l'article princeps consacré à la CIMU, Taboulet et al. [7] avaient également montré que leur échelle avait une reproductibilité forte, évaluée avec un kappa pondéré de 0,77 (ICs $95 \%$ [0,71$0,82])$. Ces résultats montraient la qualité de cette échelle, mais ils avaient été obtenus dans le berceau de la CIMU, c'est-à-dire l'hôpital Saint-Louis (AP-HP). Développé par une seule SU qui admettait à l'époque moins de 100 patients par jour, l'adaptation de la CIMU à d'autres services peut se discuter. Grâce à l'étude de l'hôpital Lariboisière, la validité externe des bons résultats attribués à la CIMU s'en trouve renforcée. Cependant, il reste encore plusieurs étapes avant de pouvoir valider une échelle de triage française au même niveau de preuve que l'emergency severity index américaine ou l'échelle canadienne de triage et de gravité. D'autres études, multicentriques avec un important effectif, devront confirmer ses reproductibilités intra- et interindividuelles, ainsi que son exactitude. Elle devra également prendre en compte les spécificités du triage pédiatrique $[8,9]$, ce que ne fait pas la CIMU, et de la médecine préhospitalière. La médecine d'urgence française a besoin d'une échelle de triage validée qui lui corresponde. 
Liens d'intérêts : Les auteurs déclarent ne pas avoir de lien d'intérêt

\section{Références}

1. Claret PG, Segal N, Maignan M, et al (2014) Le triage en structure des urgences. Ann Fr Med Urg 4:196-200

2. McHugh M, Tanabe P, McClelland M, et al (2012) More patients are triaged using the Emergency Severity Index than any other triage acuity system in the United States. Acad Emerg Med 19:106-9

3. Christ M, Grossmann F, Winter D, et al (2010) Modern triage in the emergency department. Dtsch Arztebl Int 107:892-8

4. Travers DA, Waller AE, Bowling JM, et al (2002) Five-level triage system more effective than three-level in tertiary emergency department. J Emerg Nurs 28:395-400
5. Wuerz RC, Milne LW, Eitel DR, et al (2000) Reliability and validity of a new five-level triage instrument. Acad Emerg Med 7:236-42

6. Fehre A, Chauvin A, Durand-Stocco C, et al (2015) Évaluation de l'application d'un triage par la Classification Infirmière des Malades aux Urgences par des infirmiers organisateurs de l'accueil en comparaison avec un triage réalisé par un médecin. Ann Fr Med Urg 5:150-8

7. Taboulet P, Moreira V, Haas L, et al (2009) Triage with the French Emergency Nurses Classification in Hospital scale: reliability and validity. Eur J Emerg Med 16:61-7

8. Cheron G, Angoulvant F (2015) Triage aux urgences pédiatriques : où en sommes-nous? Ann Fr Med Urg 5:1-2

9. Courtois E, Carbajal R, Galeotti C (2015) Enquête nationale sur les méthodes de triage aux urgences pédiatriques. Ann Fr Med Urg 5:3-8 